\title{
Characterizing Atomic Ordering in Intermetallic Compounds Using X-ray Energy Dispersive Spectroscopy in an Aberration-Corrected (S)TEM
}

\author{
Robert Williams ${ }^{1}$, Anna Carlsson ${ }^{2}$, Arda Genç ${ }^{3}$, John Sosa ${ }^{1}$, David McComb ${ }^{1}$ and Hamish Fraser ${ }^{1}$ \\ 1. Center for the Accelerated Maturation of Materials. Department of Materials Science and Engineering, \\ The Ohio State University, 1305 Kinnear Road, Columbus, OH USA \\ 2. FEI Company, Achtseweg Noord 5, Eindhoven, The Netherlands \\ 3. FEI Company, 5350 NE Dawson Creek Drive, Hillsboro, Oregon
}

Intermetallic compounds have been the subject of considerable interest as structural and functional materials in a wide range of applications. They are used as engineered materials themselves or as second phase components in high performance applications. It is important that these materials be fully characterized to permit effective alloy development to meet the requisite balance of properties for a given application. One important parameter is the degree to which these compounds are ordered. This is particularly important in a new series of materials known as high entropy alloys (HEA), or compositionally complex alloys (CCA), which contain typically four to six alloying elements, each at or near to equi-atomic concentrations. Many of these CCAs contain an intimate mixture of a disordered bcc phase and an ordered phase with the B2 crystal structure. These ordered phases contain reasonable concentrations of many of the alloying elements in the alloy, and so it is of interest to know how the elements are partitioned to the two sublattices in the ordered structure and to what degree anti-site defects are tolerated, i.e., what is the degree of order in these compounds. An approach to the determination of this latter parameter has been afforded by the combination of aberration-corrected S(TEM) instruments, where electron probe sizes less than interatomic spacings may be achieved coupled with X-ray energy dispersive spectrometers (XEDS) making use of silicon drift detectors(SDD) and large collection angles. This approach has been adopted in the present research, making use of an FEI Themis ${ }^{\mathrm{TM}}$ instrument equipped with Super-X $\mathrm{X}^{\mathrm{TM}}$ XEDS. Experimentally, the compositions of the individual sublattices are determined from spatially-resolved XEDS measurements and subsequent data analysis, and these compositions are plotted onto an Ordering Tie-Line diagram[1], from which the degree of order is deduced.

Effort was focused on the determination of the degree of order of the B2 phase present in the CCA $\mathrm{CoCrCuFeNiAl}{ }_{1.5}$. Initially, however, a "standard" sample was employed, namely the compound NiAl, where the sublattice occupancy is well known. This talk will present the results of applying the approach described above to the determination of the degree of order of the $\mathrm{B} 2$ compound $\mathrm{NiAl}$, and also the ordered B2 phase in the CCA. Account is taken of the expected electron delocalization and also spectral contributions from phonon scattered electrons, which was done using the procedures described by Allen and co-workers [ref]. More serious difficulties were encountered when using Gaussian functions to effect extraction of integrated intensities from maxima, located at the positions of the atomic columns, in the signal profiles derived from the XEDS data. The custom of selecting the "best fit" for the Gaussian functions did not yield a unique solution. Possible solutions to this problem will be discussed, and an assessment of the degree to which these permit a quantitative determination of the degree of order will be presented.

\section{References:}


[1] D.H. Hou, I.P. Jones and K.L. Fraser, The Ordering Tie-Line Method for Sublattice Occupancy in Intermetallic Compounds, 1996, Phil. Mag., A74, 741.

[2] B. Forbes et al, The Contribution of Thermally Scattered Electron to Atomic Resolution Maps, 2012, Phys. Rev. B, 86, 024108.

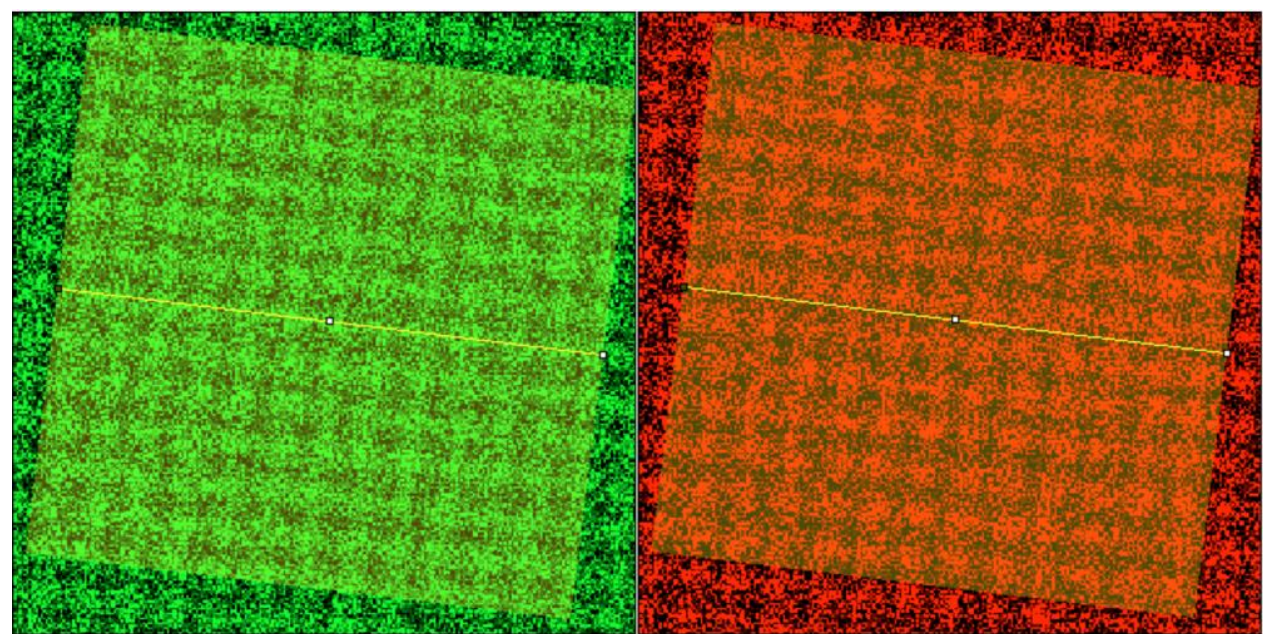

Figure 1: Raw, unfiltered XEDS map indicating $\mathrm{Ni}$ and $\mathrm{Al}$ concentrations on an apparent atomic resolution level. Yellow line indicates the direction of the line profile and the yellow box indicates the region of integration to achieve better signal to noise for quantification.
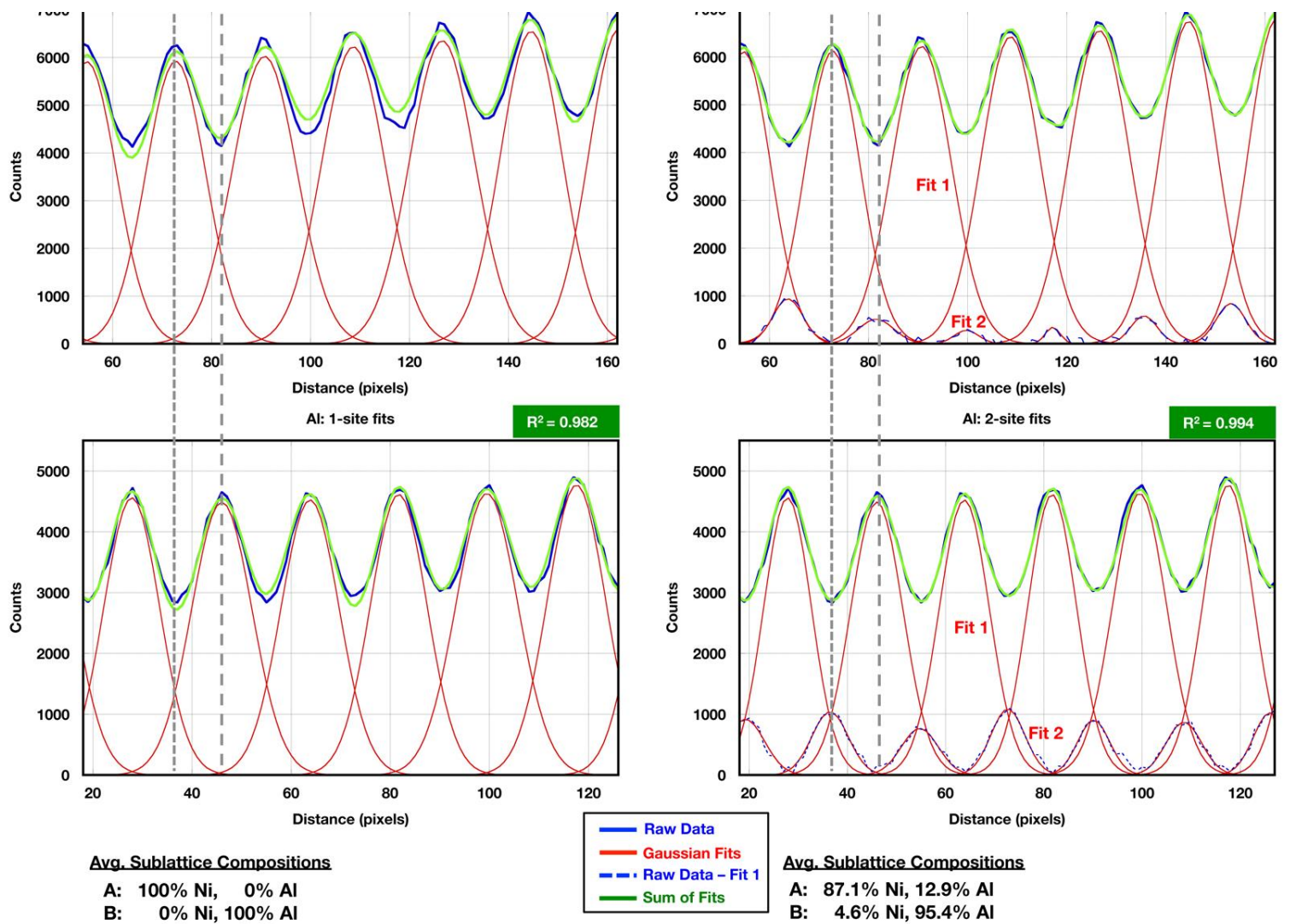

Figure 2: Guassian fitting to integrated line profiles extracted from the spectrum images shown in Figure 1. The optimal $\mathrm{R}^{2}$ value does not return the extensively characterized stoichiometry of NiAl. 